\title{
INVESTIGACIONES
}

\section{Pasantía al extranjero: estrategia para el desarrollo de la competencia intercultural en la formación inicial docente}

\author{
Short Internship Abroad: Strategy for the Development \\ of Intercultural Competence in Teacher Training
}

\author{
Eladio Donoso ${ }^{a}$, Liza Pérez ${ }^{a}$, Brandon Luna $^{a}$, Nikol Jorquera $^{a}$ \\ ${ }^{a}$ Escuela de Inglés, Universidad Católica del Norte. \\ edonoso@ucn.cl, lperez@ucn.cl,brandonluna013@gmail.com,nikoljorqueramarchant@gmail.com
}

\begin{abstract}
RESUMEN
El fenómeno de inmigración ha tenido un impacto significativo en las aulas chilenas. Ante esto se observa que los profesores no han sido preparados en su formación inicial docente (FID) para trabajar con estudiantes culturalmente heterogéneos. En este artículo se presenta un estudio de casos cuyo objetivo fue determinar si la experiencia de una pasantía corta en el extranjero reporta beneficios en el desarrollo de la competencia intercultural durante la FID de tres estudiantes de pedagogía de una universidad ubicada en el norte de Chile. Desde un enfoque cualitativo, la información fue recabada a través de entrevistas semiestructuradas. Los resultados permiten sostener que la experiencia de la pasantía fue ventajosa para los participantes y por lo tanto se propone como una estrategia FID para el desarrollo de dicha competencia.
\end{abstract}

Palabras claves: formación inicial docente en Chile, interculturalidad, competencia intercultural, pasantía.

\begin{abstract}
The phenomenon of immigration has had a significant impact on Chilean classrooms. Given this, it is observed that teachers have not been prepared in their initial teacher training (ITT) to work with culturally heterogeneous students. This article presents a case study whose objective was to determine if the experience of a short internship abroad provides benefits in the development of intercultural competence during the ITT of three students of pedagogy from a university located in the north of Chile. From a qualitative approach, the information was collected through semi-structured interviews. The results allow sustaining that the experience of the internship was advantageous for the participants and therefore it is proposed as an ITT strategy for the development of such competence.
\end{abstract}

Key words: initial teacher education in Chile, cross-culture, cross-cultural competence, internship. 


\section{INTRODUCCIÓN}

Actualmente existe un aumento significativo en la población de inmigrantes que se ha establecido en Chile. Según Rojas y Silva (2016), el porcentaje de población migrante se duplicó: en 1982 el ingreso de inmigrantes marcó un 0.7\%, mientras que el año 2015 el ingreso fue $2.3 \%$.

Lo anterior se refleja particularmente en el ámbito de la educación, produciendo un cambio significativo en las aulas chilenas. A este respecto, el Ministerio de Educación (2017) informa, por ejemplo, que en la Región de Antofagasta el 14,2\% de los estudiantes son extranjeros. Este fenómeno trae consigo varios e interesantes desafíos tanto para docentes como directivos y la comunidad escolar toda. Uno de estos retos es el fenómeno de la interculturalidad, lo que, de acuerdo con García Plaza y Oliveras Busquets (1999) hace que muchos docentes advierten nuevas necesidades formativas, imprescindibles para poder enfrentarse con éxito al multiculturalismo en la sala de clases. Es así que se hace indispensable incorporar en el currículo de la Formación Inicial Docente (FID), elementos que conlleven al desarrollo lo que se denomina competencia intercultural (Deardorff, 2004, 2009).

En cuanto a lo anterior, Sánchez, Navas y Holgado (2013, p. 241) sostienen que:

El sistema de educación superior chileno parece permanecer ajeno a estas demandas de formación o, al menos, eso parece desprenderse del hecho que no se considere explícitamente necesario incluir en los programas de formación de profesores, una educación sistemática que permita a los futuros maestros capacitarse para atender las conductas diferentes, derivadas de adecuaciones culturales diversas, y aproximarse con menos prejuicios al alumnado con condicionantes-culturales diferentes.

Se confirma, de esta manera, que el profesorado chileno está acostumbrado a una educación monocultural y que, en el desarrollo de su carrera inicial docente, fue preparado para enfrentar un alumnado homogéneo. Ello explicaría que algunos profesores se vean sorprendidos tanto por el ingreso de estudiantes extranjeros en sus centros educativos, así como por las escasas herramientas de formación profesional con que cuentan para asumir el desafío de educar desde la multiculturalidad (Barrios-Valenzuela y Palou-Julián, 2014).

Pues bien, el estudio que se reporta en este artículo es un estudio de casos, el que explora el posible beneficio de una experiencia de pasantía al extranjero, específicamente en colegios de Puerto Rico, por parte de tres futuros profesores pertenecientes a una universidad chilena situada en el norte del país. Con ello se espera establecer si esta actividad contribuye al desarrollo de la competencia intercultural.

\section{INTERCULTURALIDAD}

El término interculturalidad es más bien analítico y multidimensional puesto que se aborda desde distintas perspectivas como la ética-filosófica, epistémica, pedagógica, metodológica, social, política y jurídica (Di Caudo, Llanos Erazo y Ospina, 2016).

Desde la pedagogía, el término interculturalidad, junto con el de multiculturalismo, son las dos principales teorías que abordan, desde distintos ángulos, las problemáticas e incertidumbres que asoman desde de la diversidad cultural (Stefoni, Stang y Riedemann, 2016). 
A partir de una perspectiva amplia, el multiculturalismo es reconocer plenamente el carácter multilingüe, multiétnico y pluricultural de un país o nación, lo que genera políticas públicas a todo nivel (Salazar Tetzagüic, 2009). De esta manera, el autor enfatiza "El multiculturalismo se apoya en valores como la equidad y la justicia, la igualdad con derecho a la diferencia étnica y cultural, y se manifiesta a través del ejercicio de reconocimiento, respeto y promoción de la identidad y cultural y lingüística" (Salazar Tetzagüic, 2009, p. 15).

Por su parte, la interculturalidad, que tiene su origen en la filosofía del pluralismo cultural, se centra su atención en la interacción de las culturas, enfatizando, más que las diferencias, las convergencias que contribuyen a la convivencia aportando en valores, identidad y desarrollo (Salazar Tetzagüic, 2009). De igual forma, la UNESCO (2005, p. 5) ya definía la interculturalidad como "la presencia e interacción equitativa de diversas culturas y la posibilidad de generar expresiones culturales compartidas, adquiridas por medio del diálogo y de una actitud de respeto mutuo".

El término interacción es el que determina la conceptualización de la interculturalidad. Esta interacción es, entre otros, de carácter, lingüístico, étnico, religioso, y se caracteriza, de acuerdo a Giménez (2000) por la confianza, reconocimiento mutuo, comunicación efectiva, diálogo y debate, aprendizaje mutuo, intercambio positivo, resolución pacífica del conflicto, cooperación y convivencia. Al respecto el autor sostiene:

De todas ellas son esenciales el reconocimiento mutuo, intercambio y la convivencia, como realmente definitorias de que se está en relación de interculturalidad, para que se puedan dar, es preciso que estén también presentes las otras. La confianza, la comunicación efectiva o el aprendizaje mutuo son, por supuesto, condiciones sintomáticas de y necesarias para la interculturalidad, pero no son en sí mismas suficientes, mientras que consideramos que el reconocimiento mutuo, el intercambio positivo y la convivencia social, entendidas tal y como señalamos más adelante, son ya condiciones suficientes (Giménez, 2000, pp. 36-37).

En el ámbito de la educación, específicamente para el profesor o la profesora, la interculturalidad implica muchas cosas. Giménez (2003, p. 20) resalta algunas:

...trabajarse la tolerancia activa (en el campo de los valores), superar el etnocentrismo (en el campo de las actitudes), saber escuchar más atentamente al Otro y preguntarle con interés antes de hablar (en el campo de las habilidades) y un intento por disfrutar la diversidad profunda de su aula, escuela y mundo, a pesar de todas las dificultades de comunicación, falta de tiempo, recursos, etc. (en cuanto a su modo cotidiano de ejercer su oficio).

En este sentido, y tal como lo sostienen Figueredo Canosa y Ortiz Jiménez (2014), es importante, desde la FID y formación continua, que se promueva la adquisición de las competencias necesarias para dar respuesta a los retos que supone una educación intercultural. 


\section{COMPETENCIA INTERCULTURAL}

Como punto de partida, debemos apropiarnos del término competencia en el contexto de este estudio. Para ello adherimos a lo que sostiene Valverde López (2010) en relación al desarrollo de competencias culturales. El autor señala que competencias "Son aquellas que hacen capaz a un individuo para la realización de determinadas actividades en diferentes ámbitos, y que se derivan de la posesión de conocimientos, habilidades, destrezas, hábitos, actitudes y valores" (Valverde López, 2010, p. 141).

Las aproximaciones para definir competencia intercultural son múltiples, sin embargo, convergen en cuanto a que destacan una amalgama de conocimiento, actitudes y habilidades. Es así que para Deardorff (2006, p. 247) la competencia intercultural se define como "the ability to communicate effectively and appropriately in intercultural situations based on one's intercultural knowledge, skills, and attitudes". De igual forma, para Milstein, Otaso y Fuks (2016, p. 247) la competencia intercultural es la "que combina conocimiento de ciertos grupos culturales junto con actitudes y habilidades para tratar con la diversidad cultural".

Específicamente en el ámbito de la educación, el término competencia intercultural, se ha asociado, según un estudio de la investigadora estadunidense Deardorff (2004), a más de diez conceptos sinónimos. Estos son adaptación cultural, sensibilidad intercultural, competencia multicultural, competencia transcultural, efectividad transcultural (crosscultural effectiveness), competencia internacional, alfabetización global (global literacy), ciudadanía global (global citizenship), competencia cultural, y cambio transcultural (cross-cultural adjustment). No obstante lo anterior, el término competencia intercultural sigue siendo el más apropiado en este ámbito según la literatura reciente.

Ahora bien, Figueredo Canosa y Ortiz Jiménez (2014), basándose en numerosos estudios de diferentes autores, presentan tres dimensiones de la labor docente desde la perspectiva intercultural: cognitiva, técnica y actitudinal. Respecto de estas dimensiones, los autores definen lo siguiente:

Dimensión cognitiva de la competencia intercultural: hace referencia al conocimiento y comprensión [de] las diferencias culturales y, cómo influyen en la forma de pensar y actuar de las personas. Permite modificar concepciones erróneas que normalmente conllevan la asunción de estereotipos y prejuicios conducentes a la discriminación. (Figueredo Canosa y Ortiz Jiménez, 2014, p. 164).

Dimensión técnica de la competencia intercultural: alude a las habilidades y capacidades para gestionar las interacciones e intercambios entre las diferentes culturas logrando el enriquecimiento cultural personal y colectivo. Facilita al profesorado la construcción de un clima de convivencia en el aula y el centro, favoreciendo la inclusión de todo el alumnado. (Figueredo Canosa y Ortiz Jiménez, 2014, p. 164).

Dimensión actitudinal de la competencia intercultural: apunta a una forma de ser y actuar determinada que favorece y promueve el respeto, la aceptación y la valoración de las diferencias culturales. (Figueredo Canosa y Ortiz Jiménez, 2014, p. 165).

Estas tres dimensiones de la competencia intercultural deberían ser tomadas en cuenta y dominadas por los profesores en su labor profesional para atender las necesidades de sus estudiantes en el aula. 


\section{FORMACIÓN INICIAL DOCENTE Y COMPETENCIA INTERCULTURAL}

De acuerdo con lo planteado hasta aquí, ser interculturalmente competente es indispensable en el contexto educativo de hoy. Es así que el desarrollo de dicha competencia debe ser un elemento clave en la FID en nuestro país. La interrogante que surge es cómo la FID en las instituciones con programas de pedagogías aborda la interculturalidad y si éstas se ocupan de desarrollar la competencia intercultural.

Respecto a lo anterior, hace más de dos décadas Tabachnick y Zeichner (1993) dan cuenta de tres alternativas que parecen caracterizar a las instituciones y ello, al parecer, como se verá más adelante, no ha cambiado mucho. Los autores sostienen que la primera opción es ignorar la diversidad y mantener el statu quo, es decir, preparar a los futuros profesores para atender estudiantes homogéneos, sin tomar en cuenta la diversidad que pueda encontrar en el aula. La segunda es una alternativa que presta mínima atención al fenómeno, agregando en el currículo solamente algunos temas. Esto demuestra que la atención a la diversidad cultural aún no es importante en la FID. Por último, es una postura ideal, en donde los énfasis de la FID están puestos en desarrollar sensibilidad ante la diversidad, preparando así a los profesores en formación para enfrentarse idóneamente para su futuro desempeño profesional. Compartimos con Tabachnick y Zeichner (1993), que la tercera alternativa es la deseable por parte de las instituciones FID.

En cuanto a lo anterior y poniendo nuestra atención en Chile, en un estudio a partir de aportes teóricos recogidos en exploraciones previas, Aranda (2011) plantea la necesidad de una educación intercultural en la FID como solución a problemáticas tales como el racismo, xenofobia y la discriminación. Es por ello que el estudio de Morales, Sanhueza, Friz y Riquelme (2017) resulta de alto interés. Los autores evalúan la sensibilidad que posee el profesorado que trabaja en aulas culturalmente diversas a partir de las variables de género, edad, cursos de perfeccionamiento y experiencia intercultural que caracterizaban a los sujetos investigados, 50 profesores de 5 centros educativos con una alta población multicultural. Los resultados indican que el profesorado posee interés en conocer y compartir con personas de otras culturas, sin embargo, a estos profesores les incomoda relacionarse e interactuar con dichas personas.

En esta misma línea, Jiménez Vargas y Montecinos Sanhueza (2018) sostienen que pese a la heterogeneidad que caracteriza a las escuelas actualmente y pese a que estudios han revelado la falta de competencias por parte de los profesores y profesoras para atender a esta realidad, "los programas de formación docente en Chile no han respondido oportunamente a esta nueva problemática educativa." (Jiménez Vargas y Montecinos Sanhueza, 2018, p. 4). Sostienen además que estudios "han evidenciado que, tanto desde el punto de vista de los planes de estudio, como de la percepción del profesorado egresado, las instancias de formación destinadas a abordar la diversidad son escasas." (Jiménez Vargas y Montecinos Sanhueza, 2018, p. 4).

A partir de lo hasta aquí expuesto, se puede argumentar que es altamente necesario incorporar, de manera decidida al currículo de las pedagogías, actividades estratégicas que les permitan a los futuros profesores desarrollar la competencia intercultural. En este contexto, resulta interesante observar lo que propone Deardorff (2011). La autora sugiere tres posibilidades para desarrollar en los estudiantes terciarios la competencia intercultural (no necesariamente para futuros profesores) sobre la cual sostiene "the term used will be intercultural competence, given that it applies to any who interact with those from different 
backgrounds, regardless of location" Deardorff (2011, p. 66). Estas tres posibilidades o métodos son, en primer lugar, el aprendizaje por servicio (service learning) el que se desarrolla en un contexto doméstico y voluntario en cual el estudiante es enfrentado a diferentes ambientes culturales, promoviendo así una reflexión crítica, lo que les permite aprender y entender su propia identidad y la identidad de los otros. El segundo método es la internacionalización en casa (internationalization at home) en el propio campus universitario. Esto involucra la maximización de los recursos multiculturales disponibles en el plantel universitario para promover las oportunidades de aprendizaje intercultural. Por último, los programas de estudio en el extranjero (study abroad), lo que involucra una adecuada preparación que les permita articular nuevos aprendizajes que vayan más allá de una mera experiencia internacional. Todo lo anterior, según Deardorff (2011) puede causar una positiva transformación y apertura de los estudiantes.

Es por lo anterior que este estudio, como ya se ha mencionado, investiga sobre los posibles beneficios de una experiencia de una pasantía en el extranjero o pasantía internacional como estrategia para el desarrollo de la competencia intercultural en la FID. Se entiende como pasantía a una actividad o trabajo educativo, pues es de naturaleza académica y, por lo tanto, no son parte del trabajo asalariado tradicional (Domínguez Pachón, 2009) y su fin es facilitar la articulación entre habilidades, el conocimiento y valores, componentes esenciales de una competencia. Ahora bien, si la pasantía es en el extranjero, se añade el componente cultural, desarraigando al/la pasante de su cotidianidad, exponiéndolo/la a contextos nuevos y diferentes, lo que debería redundar en una reflexión aún más profunda sobre su propio proceder.

\section{METODOLOGÍA}

El trabajo que se reporta en este artículo es un estudio de casos, de carácter exploratorio y de tipo hermenéutico. De acuerdo con Cárcamo (2005, p. 202), “el análisis hermenéutico se enmarca en el paradigma interpretativo comprensivo; lo que supone un rescate de los elementos del sujeto por sobre aquellos hechos externos a él. En este sentido, debe destacarse que dicho análisis toma como eje fundamental el proceso de interpretación." Bajo esta perspectiva, se establecen los siguientes objetivos de investigación:

1. Obtener información sobre la experiencia de pasantía al extranjero de tres estudiantes de pedagogía de una universidad chilena situada en el norte del país. Esto a un año de ocurrida la experiencia.

2. Establecer si los sujetos aprecian como beneficiosa (o no) la experiencia de la pasantía al extranjero en su formación profesional como profesores en vista del desarrollo de su competencia intercultural.

Con el fin de contextualizar la experiencia de pasantía en el extranjero, ha de informase que los tres sujetos de este estudio de casos se sometieron a dos fases. La primera fue una de carácter preparatorio, durante la cual se sometieron a un tutelaje. Aquí se les proveyó de información sobre las características culturales del país anfitrión, Puerto Rico, y de su sistema educacional. De igual forma se reflexionó sobre conceptos como cultura, etnocentrismo, estereotipos, entre otros. La segunda fase consistió en la 
experiencia misma, es decir, las actividades tanto en los colegios como en la comunidad en donde estaban situados los colegios en Puerto Rico. Esta fase tuvo una duración de un mes. Las actividades fueron acompañadas de reflexiones diarias las que los pasantes debían consignar en bitácoras o diarios personales y, luego, compartirlas entre ellos y la académica que los acompañó.

Lo anterior ha sido el insumo para poder recabar información y así llevar a cabo este estudio de caso. Pues bien, la recolección de los datos se realizó mediante entrevistas semiestructuradas con los tres sujetos seleccionados, instrumento que por sus características favoreció el acceso a sus percepciones e interpretaciones respecto a lo que ha significado para ellos haber participado en la pasantía en relación con su FID. El guion de la entrevista incorporó preguntas iniciales para adentrarlos en el tema, para luego enfocarlas a las concernientes a los objetivos del estudio. Cabe señalar que los tres sujetos accedieron voluntariamente a participar en las entrevistas. Éstas fueron registradas en audio y luego transcritas en su totalidad, lo que permitió un apropiado acceso a los datos durante el proceso de análisis.

El análisis de los datos se realizó a través del análisis de contenido. López Noguero (2002, p. 173) sostiene que: "Con esta técnica no es el estilo del texto lo que se pretende analizar, sino las ideas expresadas en él, siendo el significado de las palabras, temas o frases lo que intenta cuantificarse." De esta manera, se pudo obtener apropiadamente la información para los propósitos del estudio.

\section{ANÁLISIS Y DISCUSIÓN DE LOS RESULTADOS}

A continuación, se presentan los resultados de cada participante, identificados con etiquetas INF1, INF 2 e INF3 para resguardar su anonimato.

\subsection{INFORMANTE $1:$ INF1}

INF1 es una ex estudiante ya graduada de la carrera de pedagogía en inglés. Tiene 23 años y actualmente se encuentra participando en un programa de enseñanza de la lengua española en una escuela del Reino Unido.

Previo a la pasantía a Puerto Rico INF1 relata que su conocimiento sobre la cultura de Puerto Rico era escaso, pero siempre motivada a conocer más sobre ella. Algo interesante que llamó fuertemente la atención a INF1 fueron las diferencias lingüísticas. La pasante alude a que a pesar de compartir la misma lengua materna, el español, las diferencias entre el español de Chile y el de Puerto Rico son altamente notorias. Esto no fue para ella una gran sorpresa ya que se había abordado durante el tutelaje.

La participante, luego de la pasantía, manifiesta a que sus conocimientos sobre la pedagogía fueron enriquecidos, especialmente con respecto a metodologías de enseñanza. Esto es así puesto que la pasantía le sirvió para ampliar su variedad metodológica y didáctica para una clase de un segundo idioma, lo que es de gran importancia en su especialidad. INF1 destaca su capacidad para aprender a partir de las diferencias y desafíos que fueron apareciendo a medida que avanzaba su pasantía.

INF1, en particular, menciona con actitud positiva el aprendizaje obtenido en la pasantía después de un año de haber ocurrido: 
Bueno, con la pasantía aprendí muchas cosas que sé que ahora me hacen una buena profesora, tomando en cuenta la calidad de mis clases ya que yo ofrezco a mis estudiantes una gran variedad de metodologías de aprendizaje, lo que las hace ricas, entretenidas, didácticas y significativas.

Se puede sostener que las pasantías no son sólo experiencias de aprendizaje, sino que también son de carácter formativo valórico ya que INF1 sostiene que: "culturalmente eliminé estereotipos". Esto último evidencia claramente que este tipo de pasantías sirve para eliminar prejuicios que se puedan tener sobre otras culturas, entender las diferentes formas de actuar y de ver el mundo.

Es importante mencionar que INF1 muestra una actitud reflexiva sobre su percepción sobre otras culturas y cambios sobre cómo enfrentarlas. A través de su experiencia como pasante, logró visibilizar las diferencias y apreciarlas, aprendió cómo hacer las mismas cosas, pero de forma diferente, lo que le ha permitido desarrollar habilidades que la hacen hoy una persona más íntegra para enfrentar su profesión y el mundo. Esto constituye un respaldo para el desarrollo de su competencia intercultural, lo que le ha valido para desempeñarse con éxito en el programa de intercambio que está realizando hoy en el Reino Unido. INF1 sostiene: “... yo ahora me encuentro en un escenario diverso y siento que este cambio lo he enfrentado muy bien gracias a que tuve una experiencia previa, en donde me enfrenté a una cultura diferente".

Esto es un resultado directo de su experiencia en Puerto Rico como pasante y le es favorable para comprender las diferencias entre su cultura y la nueva a la que está expuesta hoy. INF1 no espera ser incluida ni tampoco ser entendida por todos. Es así que señala:

... gracias a la pasantía y a esa experiencia concreta, hoy entiendo que no soy yo, es su cultura y que tengo que respetarla porque al final yo soy la que está como inmigrante y que mi misión es conocerlos a fondo, porque al conocer otra cultura puedo entender por qué su pensamiento es así o por qué actúan así...

INF1, al momento de preguntarle si ella valora más su cultura y otras culturas después la pasantía, ella destaca que el término valor no es el más indicado: "siento que cuando tú le das valor a algo es porque tú estás diciendo que algo es mejor que otro". Declara, de igual forma que, debido a la pasantía, hoy tiene mejor comprensión de las culturas, "en este tipo de experiencia creo que cuando tú vas a otra cultura no es que tú le des valor, tú le das... tú tienes un mejor entendimiento de sus raíces, de su forma de vida, de todo". En concreto, para INF1, no se trata de valorar, sino que demostrar una actitud positiva y abierta hacia la diversidad cultural: "me ayudó a entender mejor mi cultura y a entender mejor la cultura de los demás".

Podemos sostener con certeza que, para INF1, esta pasantía le permitió aprender sobre sí misma y sobre cómo tratar con personas de otras culturas, así como también adaptarse a diferentes contextos: "la pasantía me ayudó a entender mejor esta cultura, quizá asimilar mejor, adaptarme mejor".

Ahora bien, desde el punto de vista pedagógico disciplinar, INF1 pudo darse cuenta de cómo se enseña el inglés y cuál es la diferencia de metodologías entre segunda lengua y lengua extranjera: 
eh... aprender, por ejemplo, cómo mi lengua es enseñada como segunda, o sea, como lengua extranjera y entender. Si bien la pasantía fue así como relacionada con el inglés, ver ahora el español, como que tengo una idea más global de lo que es la educación y cómo se enseña una lengua extranjera.

Para enfatizar y resumir su experiencia, INF1 menciona: "creo que quizás no entendería mucho lo que estoy viviendo ahora si no hubiera vivido lo de Puerto Rico”.

\subsection{INFORMANTE 2: INF2}

INF2 es una ex estudiante de la carrera de pedagogía en inglés. Tiene 23 años y actualmente se encuentra trabajando en una escuela pública en el norte de Chile. Si bien ella había interactuado con personas de otras culturas previo a la pasantía, estas interacciones, en sus palabras, eran: “... bastante superficiales. Al cursar la carrera, también tenemos profesores que son de distintos países, como la profesora Ávila, la profesora Liza, así que, en general, de manera muy superficial". Del mismo modo, declara que su conocimiento sobre el país anfitrión era mínimo:

lo único que sabía era que es un país que en lo económico era un país que dependía de Estados Unidos y que le da mucho énfasis en inglés por el tema de esta relación que tiene con Estados Unidos, pero más allá de eso, no sabía.

No obstante, esto no fue impedimento para mostrar emoción y disposición en conocer sobre la cultura puertorriqueña: "Estaba bastante emocionada... sentía que estaba trabajando en algo que me iba a beneficiar a mí y a los chicos que les íbamos hacer clases y también a la futura generación con la que iba a trabajar".

Al consultarle sobre diferencias más notorias entre la cultura de aula chilena y la puertorriqueña, INF2 asegura que: "En Chile somos súper reservados. Somos como bien a la antigua en el sentido de que está el profesor, el pizarrón, los chicos sentados. Pero la profesora allá vino con otra metodología, bastante didáctica, con la que todos interactuamos, reflexionamos en grupos...". De igual forma, INF2 sostiene que esto fue muy significativo:

Bueno, algo que rescato harto de allá es el hecho que quiero llevarlo a la práctica acá y lo estoy tratando de hacer ahora... es la relación que existe con el profesor y los alumnos, ya que los chicos ven al profesor como un real modelo a seguir $Y$ alguien bastante interactivo, alguien bastante movido.

Destaca, pues, el rol del profesor y como es visto por sus estudiantes y añade: "Allá, los profesores que tenían eran motivados, eran interactivos para hacer las clases era muy aperrados para trabajar con los chicos".

Cuando se le preguntó a INF2 sobre si valora más su cultura y otras culturas ahora, después de la pasantía, comenta que, en su trabajo, ella se relaciona con estudiantes de diferentes contextos culturales. A modo de ilustrar, INF2 releva que le es llamativo el hecho que estudiantes migrantes en su colegio actual deben celebrar las fechas importantes para los chilenos, las que no lo son necesariamente para ellos. Afirma que: 
“...cuando abordamos las fiestas patrias, por ejemplo, los chicos de otros países no tienen idea de lo que estamos hablando, entonces incluimos mucho por ejemplo... en Chile festejamos esto ¿pero en tu país qué hacen? ...”. Lo anterior es un claro indicio que la pasantía ha ayudado a la participante a estar más consciente y abierta a las diferencias culturales de sus alumnos, aceptándolos y permitiéndoles expresarse desde su sapiencia: "Soy bastante abierta a considerar dejar todo en la sala de clase, en la preparación de cómo voy a pasar el contenido, porque uno siempre piensa en el chileno, pero ¿qué pasa con el resto?".

Con relación a su apreciación respecto a cómo le ha favorecido la pasantía, afirma:

Yo creo que me ayudó harto a desarrollar carácter. Como al principio uno siempre está bien tímida al hacer una clase porque se basa en el modelo ortodoxo del profesoralumno. Esta pasantía me ayudó mucho a desarrollar carácter y a variar en las metodologías para abarcar a todos los chicos (...) Siento que eso es muy importante ya que la pasantía te ayuda a demostrarte que tú puedes hacerlo. De hecho, puedo decir que la pasantía me mostró que yo soy capaz de ser profesora.

\subsection{INFORMANTE 3: INF3}

INF3 es un estudiante de 29 años de la carrera de Pedagogía en Enseñanza Básica que hoy en día se encuentra cursando el último semestre del programa y, además, realizado su práctica profesional. Este informante demuestra un gran interés al momento de expresar sus vivencias con respecto a la pasantía realizada ya que, al momento de ser preguntado con respecto a su conocimiento con la cultura de Puerto Rico, indica que el solo tenía conocimiento de su parte artística: “...o sea lo que cualquier persona sabe de acuerdo a la tele, la mass media".

Con relación a algún aspecto que le haya sido significativo en cuanto a su formación como profesor, INF3 comenta sobre el contraste que existe entre las metodologías enseñadas por sus docentes durante toda su formación como profesor en la enseñanza superior y lo que vivenció en Puerto Rico. Él alude a que durante su formación lo que más aprendió fue que una clase normal debe estar dividida por un inicio, desarrollo y cierre, pero durante su experiencia en la pasantía, fue capaz de fijarse que una metodología de enseñanza fue aplicar solamente la etapa de desarrollo durante una clase completa, lo que él encontró muy práctico: "...era posible desarrollar una clase muy bien, sin problemas, no impidió que los alumnos aprendieran... no sabía que eso era posible...”.

Tomando en cuenta aspectos actitudinales, INF3 recalca su interés por conocer nuevas culturas, por empatizar con ellas. Después de su experiencia en la pasantía, dice tener todas las ganas de seguir conociendo más sobre metodologías, de seguir enriqueciendo su abanico de posibilidades que lo hagan un cada día mejor profesor: "entendí que, como profesor, tengo esa labor, de enriquecerme para yo poder entregar lo mismo a mis alumnos día tras día. Imagínense en un mes aprendí muchísimo, imagínense lo que puedo aprender si repito esta experiencia". Esta evaluación positiva de su experiencia en la pasantía lo hace proponer que sus compañeros de carrera también tengan la posibilidad y la motivación de participar de programas como éste.

IFN3 rescata, sobre todo, el impacto que tuvo en su capacidad para convivir con otras culturas, para empatizar con ellas: 
tenía miedo de ser ajeno a algo, de ser yo quien fuera el extranjero. Pensé y tenía el recelo de participar única y exclusivamente por el hecho de no saber lo que me esperaba culturalmente... Bueno, esto lo digo porque no sabía el trato que iba a recibir allá.

Cabe destacar que esto alude a la predisposición que tenía el participante, ya que él relata que ha tenido la experiencia como profesor, en el contexto de un aula culturalmente diversa “...pero ahora yo ya sé lo que se siente y sé cómo actuar en una sala de clases. Gracias a esto, también puedo empatizar con los que están del otro lado. Porque sé cómo se siente ser ajeno a algo". Para INF3 la experiencia marcó un antes y un después en su formación como profesor.

En este caso el desarrollo de competencia intercultural es innegable, ya que el participante se siente mucho más cómodo al tratar con otras culturas o al verse enfrentado a situaciones en las cuáles él debe ser quien es el ejemplo ante un grupo de alumnado, haciendo así su formación pedagógica enriquecedora.

\section{CONCLUSIÓN}

Tal como se presentó en la introducción, el objetivo general del estudio que aquí se reporta fue determinar el posible beneficio de una experiencia de pasantía al extranjero. Para ello se seleccionó tres participantes, futuros profesores pertenecientes a una universidad chilena. A través de entrevistas semiestructuradas se pudo recolectar datos que permiten sostener que efectivamente esta experiencia es beneficiosa para el desarrollo de la competencia intercultural, entendida esta en los términos de Deardorff (2011).

Tal como se describe en el apartado anterior, los tres casos coinciden en que la pasantía ha sido muy beneficiosa para ellos, especialmente para alcanzar nuevos conocimientos sobre la praxis pedagógica. Comprenden que los docentes, hoy en día, deben ser capaces de sensibilizar sus acciones y poder aprender a trabajar con diferentes realidades, ya sean culturales, económicas, sociales y personales. También, les ha permitido valorar y tener una actitud positiva hacia la diversidad cultural, sensibilizarse con el otro y darse cuentas que existen distintas realidades, distintos tipos de vulnerabilidad y que tienen que aprender a trabajar con personas de otras culturas. Es por ello que actualmente están incorporando este acervo a sus prácticas pedagógicas para así aportar al cambio necesario que los profesores deben realizar. De igual forma, con esto han podido desarrollar estrategias y habilidades para enfrentar las situaciones tanto dentro del aula como fuera de ella.

Con todo lo expuesto hasta aquí, es posible sostener que la experiencia de una pasantía, como la descrita en este estudio, podría contribuir a resolver los temas planteados por Aranda (2011) Morales, Sanhueza, Friz y Riquelme (2017) Jiménez Vargas y Montecinos Sanhueza (2018), entre otros. Pues bien, sostenemos que este trabajo realiza una propuesta original para el desarrollo de la competencia intercultural de futuros profesores. Es por lo anterior que se espera que los resultados obtenidos puedan contribuir a la discusión académica en torno a mejorar la formación de profesores competentes ante los desafíos actuales de la educación en el país. 


\section{REFERENCIAS BIBLIOGRÁFICAS}

Aranda, V. (2011). Reflexión y análisis de políticas y prácticas innovadoras a la luz de las representaciones sociales y de la necesidad de una educación intercultural en la formación inicial docente. Estudios Pedagógicos, 37(2), 301-314.

Barrios-Valenzuela, Ll. A. y Palou-Julián, B. (2014). Educación intercultural en Chile: la integración del alumnado extranjero en el sistema escolar. Educación y Educadores, 17(3), 405-42.

Cárcamo, H. (2005). Hermenéutica y Análisis Cualitativo. Cinta de Moebio. Revista de Epistemología de Ciencias Sociales, 23. Consultado de https://cintademoebio.uchile.cl/index.php/CDM/article/ view/26081/27386

Deardorff, D. K. (2004). The identification and assessment of intercultural competence as a student outcome of internationalization at institutions of higher education in the United States. Unpublished doctoral dissertation, North Carolina State University, Raleigh, NC.

Deardorff, D. K. (2006). Assessing Intercultural Competence in Study Abroad Students. In Byram, M. and Feng, A. (Eds.). Living and Studying Abroad. Research and Practice, (pp. 232-256). Clevedon: Multilingual Matters.

Deardorff, D. K. (2009). Identification and assessment intercultural competence as a student outcome of internationalization. Journal of Studies in Intercultural Education, 10, 241-266.

Deardorff, D. K. (2011). Assessing Intercultural Competence. New Directions for Institutional Research, 149, 65-79.

Di Caudo, M. V., Llanos Erazo, D. y Ospina Alvarado, M. C. (Coords.) (2016). Interculturalidad y educación desde el sur. Contextos, experiencias y voces. Cuenca, Ecuador: Universidad Politécnica Salesiana.

Domínguez Pachón, M. J. (2009). Responsabilidad social universitaria. Humanismo y Trabajo Social, 8, 37-67.

Figueredo Canosa, V. \& Ortiz Jiménez, L. (2014). Desarrollo de competencias interculturales en la formación del profesorado en Andalucía. Journal for Educators, Teachers and Trainers, 5(2), 160-179.

García Plaza, M. M. y Oliveras Busquets, E. (1999) Formación del profesorado en educación intercultural: Un caso práctico. Revista Interuniversitaria de Formación del Profesorado, 36, 199-209.

Giménez, C. (2000). Guía sobre interculturalidad. Guatemala: Colección Cuadernos Q'anil, Proyecto Q'anil-PNUD, Editorial Serviprensa CA.

Giménez, C. (2003). Pluralismo, multiculturalismo e interculturalidad. Propuesta de clarificación y apuntes educativos. Revista Educación y Futuro: Revista de Investigación Aplicada y Experiencias Educativas, 8, 9-26.

Jiménez Vargas, F. y Montecinos Sanhueza, C. (2018). Diversidad, modelos de gestión y formación inicial docente: desafíos formativos desde una perspectiva de justicia social. Revista Brasileira de Educação, 23, 1-21.

López Noguero, F. (2002). El análisis de contenido como método de investigación. Revista de Educación, 2, 167-179.

Milstein, D., Otaso, A. y Fuks, A. (2016). Interculturalidad y salud en la educación médica. En M. V. Di Caudo, D. Llanos Erazo y M. C. Ospina Alvarado (Coordinadores). Interculturalidad y educación desde el sur. Contextos, experiencias y voces (pp. 147-167). Ecuador: Universidad Politécnica Salesiana.

Ministerio de Educación. Centro de Estudios, Unidad de Estadística. (2017). Estadísticas de la educación 2016. Santiago, Chile: Autor.

Morales, K., Sanhueza, S., Friz, M. y Riquelme, P. (2017). Sensibilidad intercultural de profesores chilenos que atienden a población escolar inmigrante. Journal of New Approaches in Educational Research, 6(1), 71-77. 
Rojas, N. y Silva, C. (2016). La migración en chile: Breve reporte y caracterización. Madrid: Observatorio Iberoamericano sobre Movilidad Humana, Migraciones y Desarrollo.

Salazar Tetzagüic, M. J. (2009). Multiculturalidad e interculturalidad en el ámbito educativo. San José, Costa Rica: Instituto Interamericano de Derechos Humanos.

Sánchez, A., Navas, L. y Holgado, P. (2013). Inmigración y educación intercultural en la formación inicial docente. Estudios Pedagógicos, 39(1), 239-251.

Stefoni, C., Stang, F. y Riedemann, A. (2016). Educación e interculturalidad en Chile: un marco para el análisis. Estudios Internacionales, 185, 153-182.

Tabachnick, R. y Zeichner, K. (1993). Preparing Teachers for Cultural Diversity. Journal of Education forTeaching, 19(4-5), 113-124.

UNESCO (2005). Convención sobre la protección y promoción de la diversidad de las expresiones culturales. París, Francia.

Valverde López, A. (2010). La formación docente para una educación intercultural en la escuela secundaria. Cuicuilco, 48, 133-147. 
